\title{
3D printing of patient-specific implants for osteochondral defects: workflow for an MRI-guided zonal design
}

\author{
David Kilian ${ }^{1} \cdot$ Philipp Sembdner $^{2} \cdot$ Henriette Bretschneider ${ }^{1,3} \cdot$ Tilman Ahlfeld $^{1} \cdot$ Lydia Mika $^{2} \cdot$ Jörg Lützner $^{3}$. \\ Stefan Holtzhausen ${ }^{2} \cdot$ Anja Lode $^{1} \cdot$ Ralph Stelzer $^{2} \cdot$ Michael Gelinsky $^{1}$ (i)
}

Received: 19 February 2021 / Accepted: 12 June 2021 / Published online: 21 July 2021

(c) The Author(s) 2021

\begin{abstract}
Magnetic resonance imaging (MRI) is a common clinical practice to visualize defects and to distinguish different tissue types and pathologies in the human body. So far, MRI data have not been used to model and generate a patient-specific design of multilayered tissue substitutes in the case of interfacial defects. For orthopedic cases that require highly individual surgical treatment, implant fabrication by additive manufacturing holds great potential. Extrusion-based techniques like 3D plotting allow the spatially defined application of several materials, as well as implementation of bioprinting strategies. With the example of a typical multi-zonal osteochondral defect in an osteochondritis dissecans (OCD) patient, this study aimed to close the technological gap between MRI analysis and the additive manufacturing process of an implant based on different biomaterial inks. A workflow was developed which covers the processing steps of MRI-based defect identification, segmentation, modeling, implant design adjustment, and implant generation. A model implant was fabricated based on two biomaterial inks with clinically relevant properties that would allow for bioprinting, the direct embedding of a patient's own cells in the printing process. As demonstrated by the geometric compatibility of the designed and fabricated model implant in a stereolithography (SLA) model of lesioned femoral condyles, a novel versatile CAD/CAM workflow was successfully established that opens up new perspectives for the treatment of multi-zonal (osteochondral) defects.
\end{abstract}

\section{Graphic abstract}

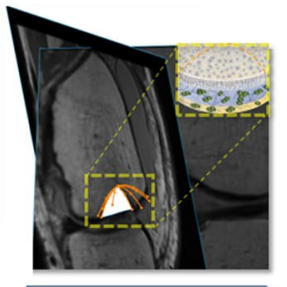

Defect

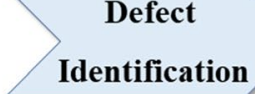

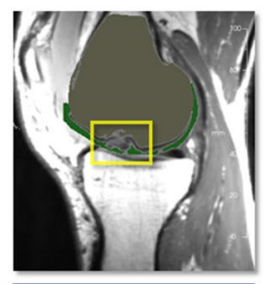

CAD

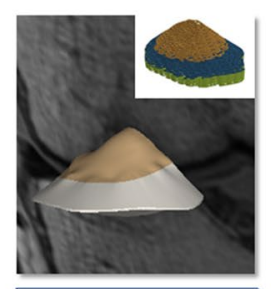

Implant

Modelling

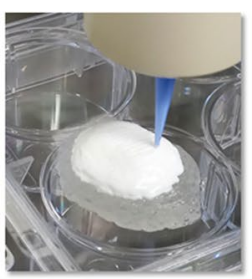

Implant

Fabrication

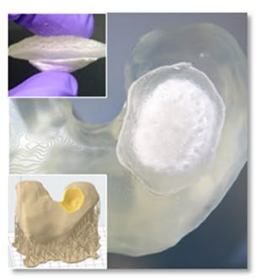

Implant

Placement

WORKFLOW FOR DESIGN AND 3D PLOTTING OF MRI-BASED OSTEOCHONDRALIMPLANTS

Keywords Additive manufacturing (AM) · Magnetic resonance imaging (MRI) $\cdot$ Computer-aided design (CAD) · Osteochondritis dissecans $(\mathrm{OCD}) \cdot$ Bone cement $\cdot$ Hydrogel

Michael Gelinsky

michael.gelinsky@tu-dresden.de

1 Centre for Translational Bone, Joint and Soft Tissue Research, University Hospital Carl Gustav Carus and Faculty of Medicine, Technische Universität Dresden, Dresden, Germany
2 Institute of Machine Elements and Machine Design, Faculty of Mechanical Engineering, Technische Universität Dresden, Dresden, Germany

3 University Center of Orthopaedic, Trauma and Plastic Surgery, University Hospital Carl Gustav Carus, Technische Universität Dresden, Dresden, Germany 


\section{Introduction}

Additive manufacturing (AM) is expected to play an essential, leading role in the field of biomedicine and for the generation of case-specific implants for all types of surgery. AM methods enable realization of a patient-specific design of implants or tissue-substituting structures. Certain techniques already allow the use of data applied for Digital Imaging and Communications in Medicine (DICOM data) from clinical imaging such as computed tomography (CT) or magnetic resonance imaging (MRI) [1] for the design of metal implants for bone replacement [2, 3]. Methods such as selective laser sintering/melting are able to consider a pre-defined individual geometry and can contribute tremendously to any biomedical application [4]. These methods and non-degradable materials play a major role in orthopedics, where the main function of surgical intervention is the mechanical support of load-sharing tissue. In addition to implant fabrication, these strategies also include the design of patient-specific molds for injection molding and individual scaffolds for tissue engineering. AM, including stereolithography (SLA), also provides new concepts for other aspects of health science-such as diagnosis, generation of surgical tools, and design of virtual and actual 3D defect models for training and patient information [5-7]. Most tissues and organs, however, consist of highly complex structures, a heterogeneous architecture, and a certain hierarchy of different cell types and anisotropic tissue zones. Therefore, computer-aided design (CAD) is based on MRI data in particular due to its better suitability with tissue characterization over CT [6]. It can contribute to highly specific, biomimetic design of tissue substitutes and scaffolds for tissue engineering, the combination of materials and (patientderived) cells [8].

Orthopedic cases often require degradable, patientspecific solutions rather than simply the purely mechanical replacement that titanium implants offer [9]. With the aid of additive manufacturing and 3D printing techniques, different material classes can play an additional role here [10-12]. With extrusion-based 3D printing (3D plotting) under mild conditions, volumetric constructs based on degradable materials can be generated with open macropores that allow infiltration with cells and nutrients (through vessel ingrowth) in order to provide oxygen and nutrients. $3 \mathrm{D}$ plotting further allows the combination of several materials through multichannel extrusion. Therefore, biomaterial inks (paste-like compositions of biocompatible materials) or bioinks - typically hydrogel blends based on (bio-)polymers with embedded living cells (3D bioprinting) — can be deposited simultaneously or independently to form individually shaped multi-material 3D constructs or implants with the potential for intrinsic biological functionality.
In orthopedic surgery, a common example of a combined (osteochondral) tissue defect in joints is osteochondritis dissecans (OCD), a defect of the subchondral bone (SCB) which also affects the covering articular cartilage (AC) [13]. About $75 \%$ of OCD cases affect the knee. However, elbow, hip, or ankle joints can be the site of impairment as well. Typically, in the initial stages OCD is primarily associated with cystic changes and bone marrow edema, caused by avascular necrosis in the bone underneath the AC layer. In severe cases and with ongoing overload, this results in "flap lesions" or fragmentation of AC ("dissection")—with free movement of tissue into the joint space. In children and adolescents, conservative therapy is often sufficient [14] while in adults the prognosis for a positive outcome is less likely [15]. In severe cases of osteochondral defect, reconstruction of the subchondral bone and cartilage is necessary [16]. Conventional strategies include retrograde drilling [17] in the early stages; and formerly, autologous cartilage implantation (ACI) [18]; matrixaugmented bone marrow stimulation in small, localized cartilage defects [19, 20]; matrix-associated autologous chondrocyte implantation (MACI) [21, 22]; or the transplantation of autologous, cylindrical osteochondral plugs from the lateral femoral condyles (FC) or bone plugs from the iliac crest [23-25] for larger defects. Some of these options indicate that tissue engineering approaches, i.e., the combination of (autologous) cells with biomaterials [26], already play a certain role which could be enhanced by means of 3D bioprinting. Compared to adolescents, the capacity for AC regeneration in adults is dramatically reduced due to senescence and the decreased number of mesenchymal stem cells available [27]. Therefore, there is a need for improvement of surgical interventions for OCD, mainly for medium and large defect sizes $\left(>3 \mathrm{~cm}^{2}\right)$, which could be accomplished by generation of multilayered tissue substitutes with an individual defect-guided design.

Although the typical in vivo resolution of CT might be better, data from CT scans are not suitable for the design of multilayered implants, including cartilage zones. In contrast to conventional CT images, MRI data can be used to identify the position, thickness, and orientation of the AC zone [28]. In this study, with the example of a patient suffering from OCD, we aimed to present how clinically obtained MRI data can be applied to help identify an individually shaped defect and then design and fabricate a multiphasic implant which fulfills clinical needs as well as the anatomical requirements of the osteochondral interface. For multichannel 3D plotting, such a workflow has not been established before. Tackling this pathological situation (Fig. 1) with the aid of design engineering methods and multichannel 3D plotting, we established a workflow integrating clinical diagnostics, tissue segmentation, 3D model generation and manufacturing 

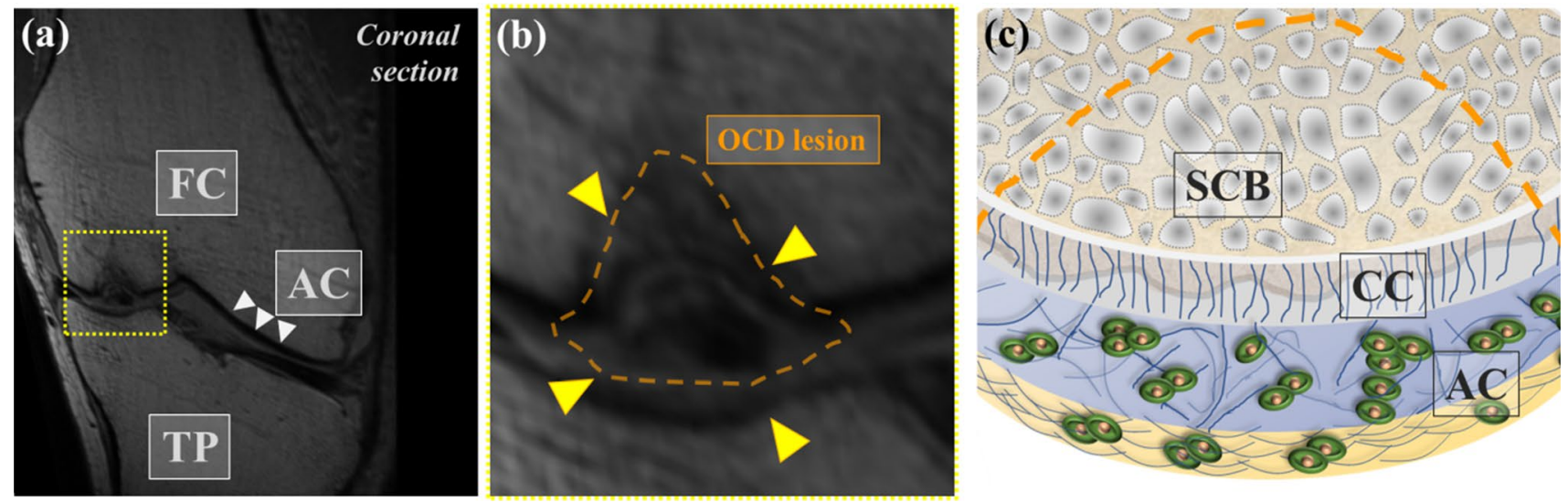

Fig. 1 Case description of the osteochondritis dissecans (OCD) pathology of a real human patient, and design process of a patientspecific tissue construct: a MRI-detected pathophysiological situation (coronal view) in the patient's defect site in the left knee, highlighted with a yellow square inside the medial femoral condyle (FC). The OCD affected both the bone zone and the articular cartilage (AC, arrows), as detected via MRI. The other side of the joint space

of a geometry-adapted, individualized, multilayered implant (Fig. 2). The implant design was intended to resemble the zonal anatomy of the osteochondral interface (Fig. 1c).

For generation of a prototype implant, we chose two model materials with the properties required for a stable zonal design. They could also be combined by multichannel plotting to generate volumetric mineralized constructs and osteochondral tissue substitutes [29]. So far, there is a limited range of hydrogels which can be combined with cells and stacked in a highly precise fashion to specific volumetric dimensions, based on different crosslinking strategies [30]. The first material was an alginate-methylcellulose (algMC) blend that was recently described for the successful encapsulation of primary human chondrocytes; it can be processed in combination with the second material, a clinically approved calcium phosphate cement (CPC), to realize interwoven and stacked multi-material scaffolds under cell-friendly conditions, with a stable interlocking effect $[29,31,32]$. The CPC is able to form is the non-affected tibial plateau (TP). b Magnified OCD lesion site, with geometry (dashed line, orange) of different affected tissue layers identified (indicated with yellow arrows). c Graphical illustration of the typical zonal anatomy of the osteochondral interface, including articular surface cartilage (AC), calcified cartilage region (CC), and subchondral bone $(\mathrm{SCB})$ region

nanocrystalline, calcium-deficient HAp closely mimicking the mineral phase of native bone, making this combination interesting for clinical application [33-36]. However, potential in vivo performance was not part of this study on geometric, MRI-based design and fabrication.

With this printable biopolymer-cement combination as the underlying concept, we developed a novel and efficient workflow (Fig. 2) for computer-aided manufacturing (CAM) that used the geometrical information obtained from MRI data. These diagnostic data were expected to be helpful for segmenting the defect site and, via implant design, 3D printing a model of a personalized tissue substitute. This workflow should be easily transferable to other material combinations besides the model system selected. In addition to commercially available software solutions, we hoped to extend the applicability of a recently developed software tool $C T$ data in CAx product development Applications (CTinA) $[3,37]$ toward use for data obtained from MRI instead of CT.

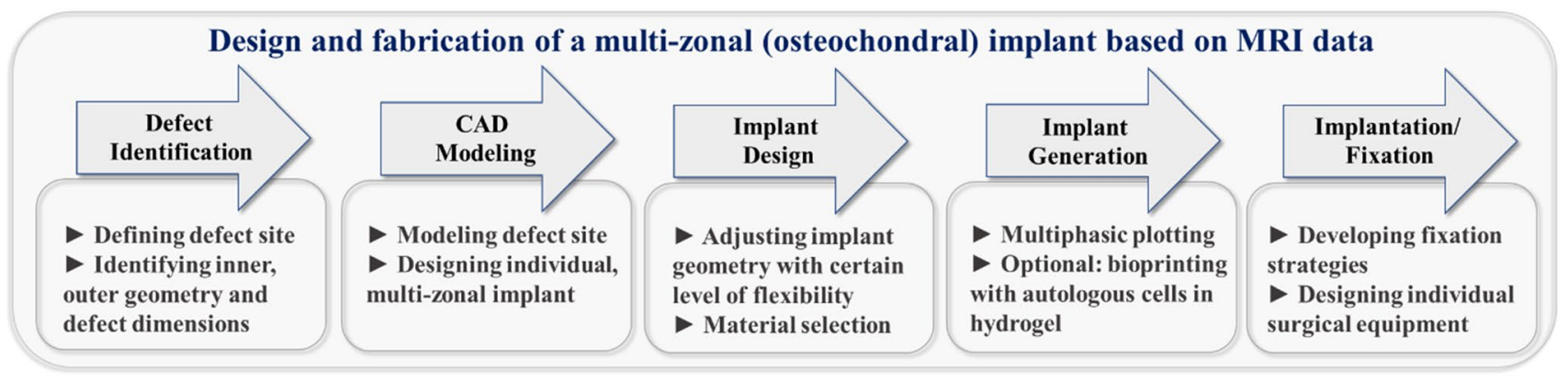

Fig. 2 Suggested workflow (with specific tasks, boxes below) for the design, fabrication, and implantation of potential multiphasic implants 


\section{Materials and methods}

\section{Properties of the underlying imaging data}

Anonymized patient data were provided after informed consent and with permission of the Ethics Committee of Technische Universität Dresden by the University Center of Orthopaedic, Trauma and Plastic Surgery, University Hospital Carl Gustav Carus, Technische Universität Dresden, Germany. MRI signals were collected using a Signa $1.5 \mathrm{~T}$ HDxt (GE Healthcare, USA) at the Institute and Polyclinic for Diagnostic and Interventional Radiology, University Hospital Dresden, Technische Universität Dresden.

$T_{1}$ sagittal and $T_{2}$ coronal sections were mainly used for identification and segmentation of the defect area, with step sizes of ca. 500 and $3500 \mu \mathrm{m}$, respectively. $T_{1^{-}}$ weighted images in the coronal and axial directions were acquired with a field of view of $90 \mathrm{~mm}$, and in the sagittal direction, with a field of view of $80 \mathrm{~mm}$. Image resolution was $512 \times 512$ pixels. In the sagittal direction, the pulse sequence parameters were determined by a repetition time of $1800 \mathrm{~ms}$ and an echo time of $25.11 \mathrm{~ms}$. In the coronal direction, repetition time was $4239 \mathrm{~ms}$ while echo time was $27.55 \mathrm{~ms}$.

\section{Identification and scaling of the defect region}

For identification and viewing of the defect site, we used a Dornheim DICOM Viewer (v2019.0.2, Dornheim Medical Images, Magdeburg, Germany). The defect region of the patient was found in the left medial femoral condyle.

We performed segmentation of the lesioned FC using Dornheim Segmenter Research (v2018.1.0, Dornheim Medical Images). For this purpose, the area of interest was manually marked in each slice image. For the bony area and cartilage area, we defined different masks to separate the structures. Automated filter algorithms such as Region Growing (for determining areas of similar gray value density based on a seed point) could not be applied due to the given conditions of the MRI dataset: An overall similar range of gray value intensities made it difficult to define thresholds for the separation of certain regions.

The segmented areas were translated into a surface model description in the Dornheim Segmenter software and finally exported as STL (stereolithography) file. Geomagic Studio 2012 software (Geomagic, Morrisville, NC, USA) was used to prepare the surface models for further processing (e.g., closing holes, smoothing). The surface model was also used to quantify the main dimensions (maximum length, width, and depth) of the identified defect region.

\section{Design of the OCD implant}

The implant geometry was first modeled with Geomagic Studio based on discrete surface data. To do this, parts of the surface were separated, offset surfaces were created and an implant model was generated using Boolean operations. We adjusted bone and cartilage zones according to the segmented defect zones. To adjust the outer AC zone of the implant, we used the intact outer line of the native AC tissue.

In addition, a new approach was introduced to directly utilize imaging data for modeling in computer-aided design (CAD) systems such as SolidWorks 2019 (Dassault Systèmes, Vélizy-Villacoublay, France). This approach, in combination with a newly developed tool (CT data in CAx product development Applications-CTinA) that was described previously [37], allows the definition of design elements (e.g., axes, planes, and splines) directly from the imaging data and direct transfer into the modeling tool. During processing, intermediate states of the modeled implant body could be visualized in CTinA with the imaging data (here MRI data) for review processes, which underlines the advantage of this bidirectional interface [37].

The main requirement for the implant design was the adjustment of the inner and outer geometry according to the MRI data. To allow a certain amount of flexibility during fitting and fixation of the implant, we chose to consider three different offset values for the inner implant-to-SCB-interface $(0.3,3$, and $5 \mathrm{~mm})$. The implant design adjustments for this study were made using Geomagic Studio. We will present SolidWorks 2019 in combination with our custom-made tool CTinA as a versatile, promising alternative path.

\section{Plotting materials}

To prepare the algMC blend, $3 \mathrm{wt} \%$ of alginic acid sodium salt from brown algae (Sigma-Aldrich, Germany) was blended with phosphate-buffered salt solution (PBS) (Thermo Fisher, USA). The homogeneous solution was autoclaved $\left(121{ }^{\circ} \mathrm{C}, 20 \mathrm{~min}\right)$ using a D-23 table-top autoclave (SysTek, Detmold, Germany). Later, $9 \mathrm{wt} \%$ of autoclaved methylcellulose powder (MC 4,000 cP, Sigma-Aldrich, USA) was added. Ink was used for 3D plotting after $2 \mathrm{~h}$ of MC swelling at RT.

As a sacrificial support, we used an ink of $10 \%$ autoclaved MC in double-deionized water, as described previously [11]. MC swelling in $\mathrm{ddH}_{2} \mathrm{O}$ was done at $4{ }^{\circ} \mathrm{C}$ overnight prior to initiating printing.

As a HAp-forming, bone-mimicking component, we chose a calcium phosphate cement (Plotter-Paste-CPC, INNOTERE, Radebeul, Germany) based on $\alpha$-TCP. 


\section{Fabrication of the implant and post-printing treatment for stabilization}

We combined the established stl files describing the geometry of the separated zones to a $3 m f$ file using 3D Builder software (Microsoft, USA). Slicing of this 3D model with pre-defined bone and cartilage zones was carried out using BioScaffolder 3.1 software (GeSiM mbH, Radeberg, Germany); the plotting process was conducted by the corresponding machine BioScaffolder 3.1. The plotting settings that we adapted for the three different materials/material blends to be extruded are summarized in Table 1 .

Strand spacing for the CPC was chosen flexibly in the range of $1.0-2.0 \mathrm{~mm}$. For the algMC ink and MC support ink, strand spacing was chosen in the range of the width of extruded strands (algMC: $500 \mu \mathrm{m}$; MC: $700 \mu \mathrm{m}$ ).

After completing the print (ca. $30 \mathrm{~min}$ ), initial setting of the CPC was possible by placing the entire implant in a $37{ }^{\circ} \mathrm{C}$ water-saturated atmosphere for $30 \mathrm{~min} .100 \mathrm{mM}$ $\mathrm{CaCl}_{2}$ solution (Sigma-Aldrich, USA) was then added for alginate crosslinking, completely covering the implant for $30 \mathrm{~min}$. By lifting up the implant in a controllable manner, a complete soaking of all parts of the implant was ensured. This two-step post-processing procedure was based on a recently established method which enables a combination with cell printing of human mesenchymal stem cells or primary human chondrocytes while maintaining cell viability and optimal CPC setting [29, 38]. In our study, after $30 \mathrm{~min}$, the entire implant was transferred to HBSS at $4{ }^{\circ} \mathrm{C}$ to speed up the dissolution process of the MC support structure. After $4 \mathrm{~h}$, the remaining MC support was removed manually.

For improved stabilization of the algMC-to-CPC interface, we incorporated six interwoven layers of this material in the most proximal part of the cartilage zone, closer to the subchondral bone phase, as described previously [38].

\section{Fitting of the generated implant into a SLA-generated femoral model}

To evaluate the fit of the generated implant into the actual defect geometry, we used the segmented FC to fabricate a 3D model of the lesioned FC and the additional AC surface. The Form 3 SLA printer (Formlabs, Boston, USA) allowed us to print a combined, monophasic model of these structures. The resolution in the z-direction was set to $100 \mu \mathrm{m}$

Table 1 Plotting parameters of implant materials and support ink

\begin{tabular}{lrrr}
\hline Plotting parameter & MC & algMC & CPC \\
\hline Nozzle diameter $(\mu \mathrm{m})$ & 610 & 410 & 410 \\
Dosing pressure $(\mathrm{kPa})$ & 300 & 90 & 130 \\
Printing velocity $\left(\mathrm{mm} \cdot \mathrm{s}^{-1}\right)$ & 8 & 8 & 7 \\
\hline
\end{tabular}

(fabrication time: $8 \mathrm{~h}$ ) after designing and adjusting an appropriate supporting scaffold structure using Preform software (Formlabs, USA). Clear Resin v4 (Formlabs, USA) was used as the photo-polymerizable resin. After fabrication, the model was washed for $10 \mathrm{~min}$ in isopropyl-alcohol (IPA) in a FormWash station (Formlabs, USA), and subsequently, after evaporation of remaining IPA, a post-curing process (for 20 min at $60{ }^{\circ} \mathrm{C}$ ) was applied with a FormCure work station (Formlabs, USA).

\section{Design and generation of patient-specific fixation equipment}

Using SolidWorks 2019 software, we designed an adapter for an implant fixation plunger with a concave surface corresponding to the outer convex shape of the cartilage zone of the implant. For this purpose, we first modeled the basic body according to the size of the implant and the given insertion tool, and then subtracted the individual outer shape of the cartilage from the model. We fabricated this model using SLA printing (Form 3; Formlabs, USA), after adjusting the related removable support structures via PreForm software (see above). High Temp Resin v2 (Formlabs, USA) was used as the photo-polymerizable material to form the adapter. After fabrication, the model was treated like the defect model (as described above) with the only difference being that the post-curing process was carried out for 120 min at $80^{\circ} \mathrm{C}$.

\section{Results}

\section{Identification, segmentation, and measurement of the lesioned region of the femoral condyles}

From the provided clinical MRI data, we were able to identify the defect region and the corresponding anatomy. No tissue fragment had detached from the FC in this case yet (OCD stage 3 ). In the sagittal slices of $T_{1}$-weighted images (Fig. 3a), we identified and segmented the FC and the articular cartilage (AC) region (Supplementary material, Fig. S1). With this, the transfer of these data to a virtual and editable environment was completed (Fig. 3), as described in "Materials and methods" section above. We then determined the maximum depth $(5.9 \mathrm{~mm})$, length $(21.7 \mathrm{~mm})$, and width (15.4 mm, Fig. 3c). For design of the corresponding implants, the base of the defect site was smoothed.

\section{Design of two implant phases for a bilayered osteochondral implant}

After identifying the defect region and determining its dimensions, we used the MRI scan to distinguish the bone 

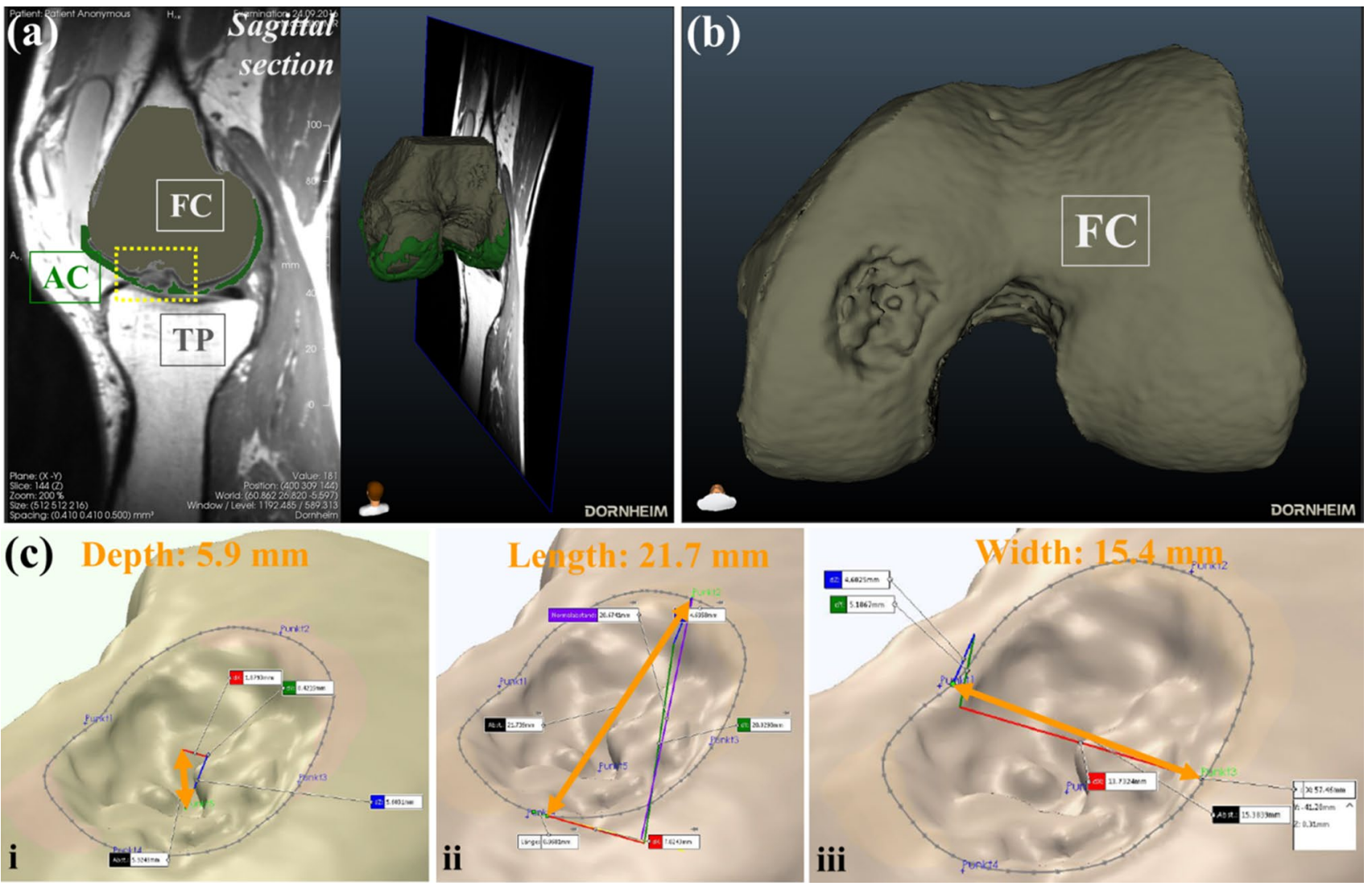

Fig. 3 Defect site modeling. a Identification, segmentation, and 3D modeling of the geometry of the lesioned femoral condyle articular cartilage (AC), separation from the surrounding muscle, tissue, and the opposing tibial plateau (TP) of the joint space, all based on MRI

and cartilage zones of the OCD defect. Here, the CTinA software tool allowed a more flexible and bidirectional workflow: It combined visualization of various orientations in the clinical imaging data with processing and volume modeling of segmented regions, and with the design of an individual implant (Fig. 4a). Based on the identified zones of damaged bone and cartilage layers, two artificial implant models were designed, smoothed, and adapted to the respective geometry (Fig. 4b i-iii). Later, we combined them to a biphasic osteochondral implant (Fig. 4b iv) that reflected the defectfacing and joint-facing geometry of the defect (Supplementary material, Fig. S2).

\section{D plotting of the biphasic osteochondral implant}

We used the generated $3 m f$ file describing the biphasic implant geometry to generate an implant based on algMC and CPC (Fig. 5), according to the pre-defined design and the layer and strand pattern obtained after slicing the 3D model (Fig. 5a). As a support structure for the overhanging algMC, we chose a dissolvable hydrogel of $10 \% \mathrm{MC}$. The original implant geometry, including the sacrificial support data. b Virtual 3D model of the damaged bone anatomy of the FC. c Measuring and determining the exact outer bone defect geometry and the maximum depth (i), length (ii), and width (iii) of the defect site based on the 3D model

material, consisted of 70 layers (Fig. 5d and 5e) or with a bone phase offset of $5 \mathrm{~mm}, 102$ layers. The completed implant was crosslinked with the above-mentioned two-step post-processing procedure, and placed in HBSS to accelerate $\mathrm{MC}$ dissolution at $4{ }^{\circ} \mathrm{C}$. The finalized implant exhibited high shape fidelity and a well-established convex shape of the outer algMC/cartilage surface, with a maximum cartilage zone thickness of 22 layers (ca. $6 \mathrm{~mm}$ ) and a minimum thickness of 12 layers (ca. $3.4 \mathrm{~mm}$ ) (Fig. 5f).

\section{Individual adjustment of the defect-facing bone phase geometry toward press-fit implantation}

For tight, stable fixation of an implant, typically a pressfit technique is applied, i.e., the implant is pushed into the defect site with strong mechanical force. Due to the diseased state of the underlying bone, the tissue generally is not solid enough to withstand the force and the weakened bone structure is pushed back. Furthermore, the CPC strands in the open-porous implant structure tend to be shifted and therefore potentially damaged. To allow some space so that the implant could be pressed into the joint without causing the 
(a)

Selective iterative query of information

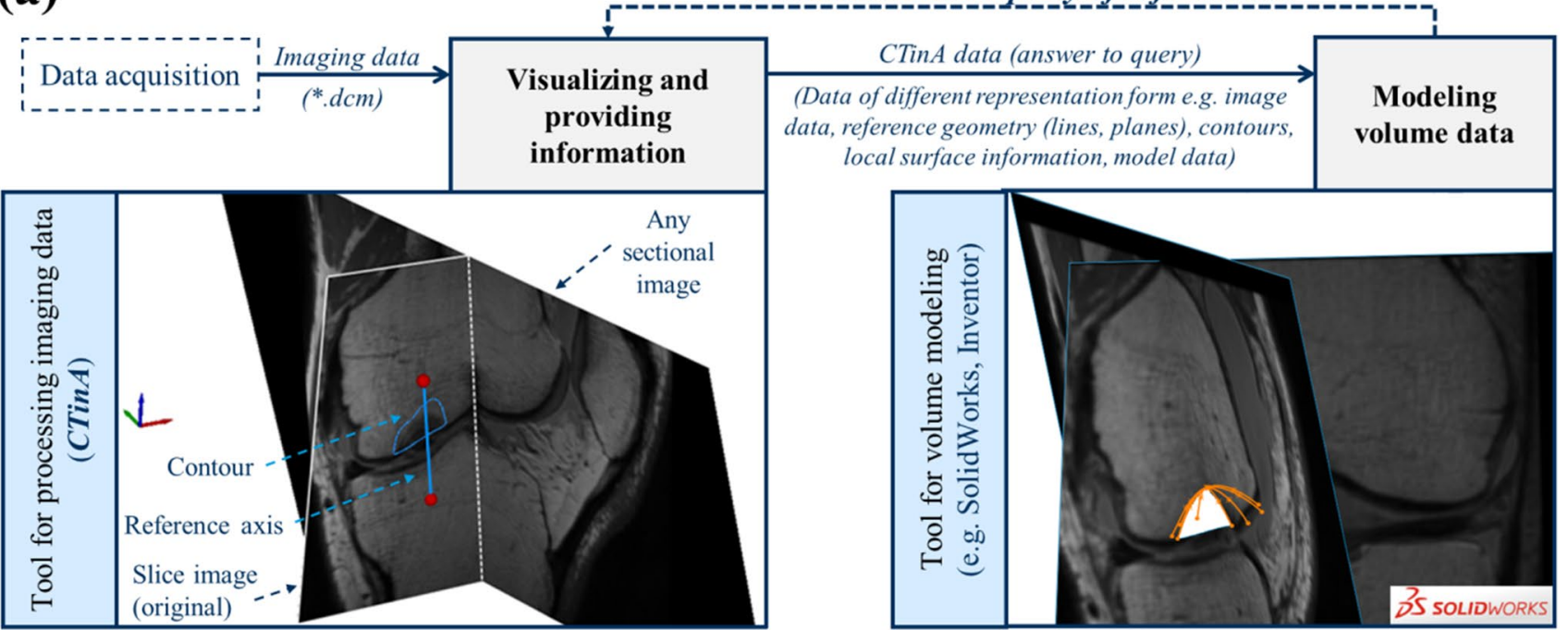

(b)
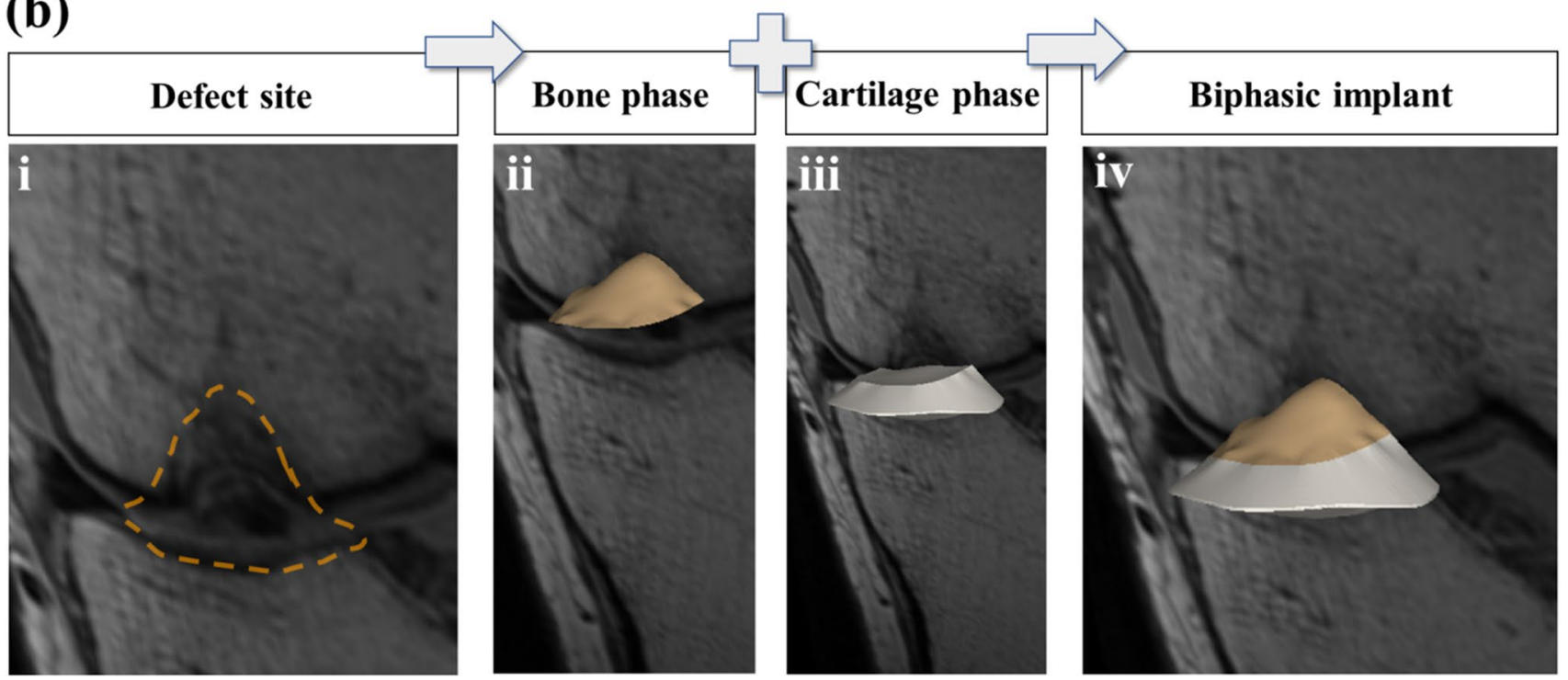

Fig. 4 a Bidirectional workflow for image-based individual design in modeling tools (left: Creation of arbitrary sectional images and references as information for modeling; right: modeling of the implant in SolidWorks based on the transferred data). b Design of the specific zones (phases) of the implant to fit the defect (orange) geometry,

risk of a misfit for the outer geometry, we adjusted the surface of the bone zone facing native tissue to allow a certain degree of flexibility during surgery. Figure 6 illustrates the accommodation for a potential shift of the internal surface that would increase the overall height of that bone phase by $0.3,3.0$, and $5.0 \mathrm{~mm}$. The geometry of the internal surface was separated from the bone-cartilage interphase and moved into the FC direction ( $z$-direction). To maintain a smooth interphase, the size of the upper geometry was slightly reduced. After establishing a new connection between the upper and lower parts, the gap between them was completed in a flat manner and smoothed.

coronal view (i). (ii) Coronal view of the osteogenic zone (beige) of the implant geometry. (iii) Convex-shaped implant zone replacing the lost cartilage layers (gray). (iv) Combining two zones into a biphasic implant, coronal view

\section{Fine-tuning of further critical design characteristics}

Besides this biphasic architecture, further crucial design characteristics are required to allow zonal fusion and appropriate in vivo integration. The fusion of the two layers/materials with long-term stability is only ensured by applying a certain number of layers of an interwoven network of the two material phases. We 3D plotted this supportive interwoven zone after adding it to the sliced model (Fig. 7a-7c). This resulted in a strong algMC-to-CPC interface adhesion that could resist external forces. Furthermore, we tuned the CPC strand spacing, and therefore the size and shape of 

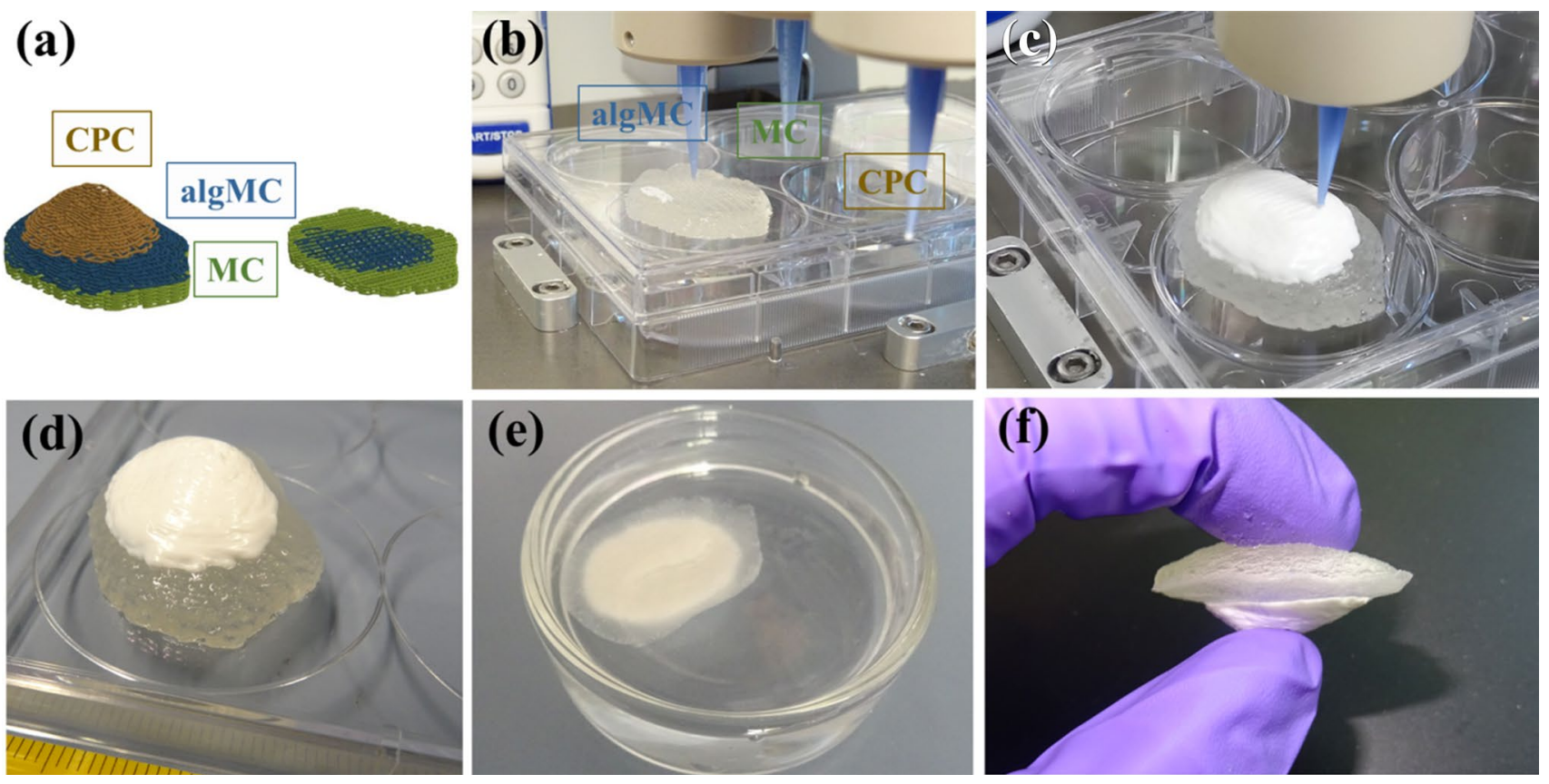

Fig. 5 Fabrication of the individual, multiphasic OCD implant with $10 \% \mathrm{MC}$ as a sacrificial support material. a Design of the sliced plotting paths (software: GeSiM BioScaffolder 3.1) for the three required materials (brown: CPC, blue: algMC, green: $10 \% \mathrm{MC}$ ), full design, and sectioned design to illustrate the need for a support material (MC, green). b Multichannel 3D plotting of the implant into the MC-based

macropores (Fig. 7d and 7e), in order to balance stability and invasion capacity for surrounding cells in vivo. This provided the required factors for flexibility, in addition to the offset of the bone geometry described above (Fig. 6).

\section{Fitting of the generated implant}

To evaluate the design and geometric compatibility of the finished implant, we generated a model of the lesioned FC combined with the articular cartilage surface via SLA, using a photo-polymerizable resin (Fig. 8). The generated implant was inserted into the lesioned site of interest (Fig. 8e) and revealed a perfect fit to the corresponding geometry. We successfully restored the outer convex shape by inserting the implant (Fig. 8g).

\section{Design and fabrication of an individualized fixation device}

To enable a smooth placement of the implant and avoid damage during the implantation procedure, we designed an individually shaped adapter for the fixation instruments, which took into consideration the outer shape of the implant surface. Based on the convex shape of the cartilage phase, we designed and fabricated a concave adapter tip for a surgical plunger via SLA (Fig. 9). This adapter cylinder, with a support structure. c Generation of the full implant. d Completed implant after plotting prior to alginate crosslinking via $\mathrm{CaCl}_{2}$, scale in $\mathrm{mm}$. e Dissolving MC support by incubation in Hank's Balanced Salt Solution (HBSS) at $4{ }^{\circ} \mathrm{C}$. f Implant structure after dissolving support material

length of $25 \mathrm{~mm}$ and an inner diameter of $8 \mathrm{~mm}$, connected the individually shaped head (thickness $5-8 \mathrm{~mm}$ ) to the surgical plunger. For orientation during surgery, anterior (A)/ posterior $(\mathrm{P})$ indications were included at the back of the adapter head.

\section{Discussion}

This study demonstrates a proof-of-concept and suggested workflow for the design, generation, and visualization of an in vitro approach for an MRI-guided, individual osteochondral implant design. The central goal was the design and generation of a multi-zonal, multi-material implant for the presented defect that would fulfill defined requirements of geometry, fabrication, stability, biological degradability, and potentially intrinsic biological functionality.

\section{A novel MRI-based workflow with the potential to overcome current clinical challenges}

The use of MRI data comes with both challenges and opportunities for workflow development: The quality of MRI data sometimes makes it difficult to define the right interfaces and geometries. Nevertheless, for the clinical diagnosis/monitoring of osteochondral lesions pre- and post-surgery, MRI is 

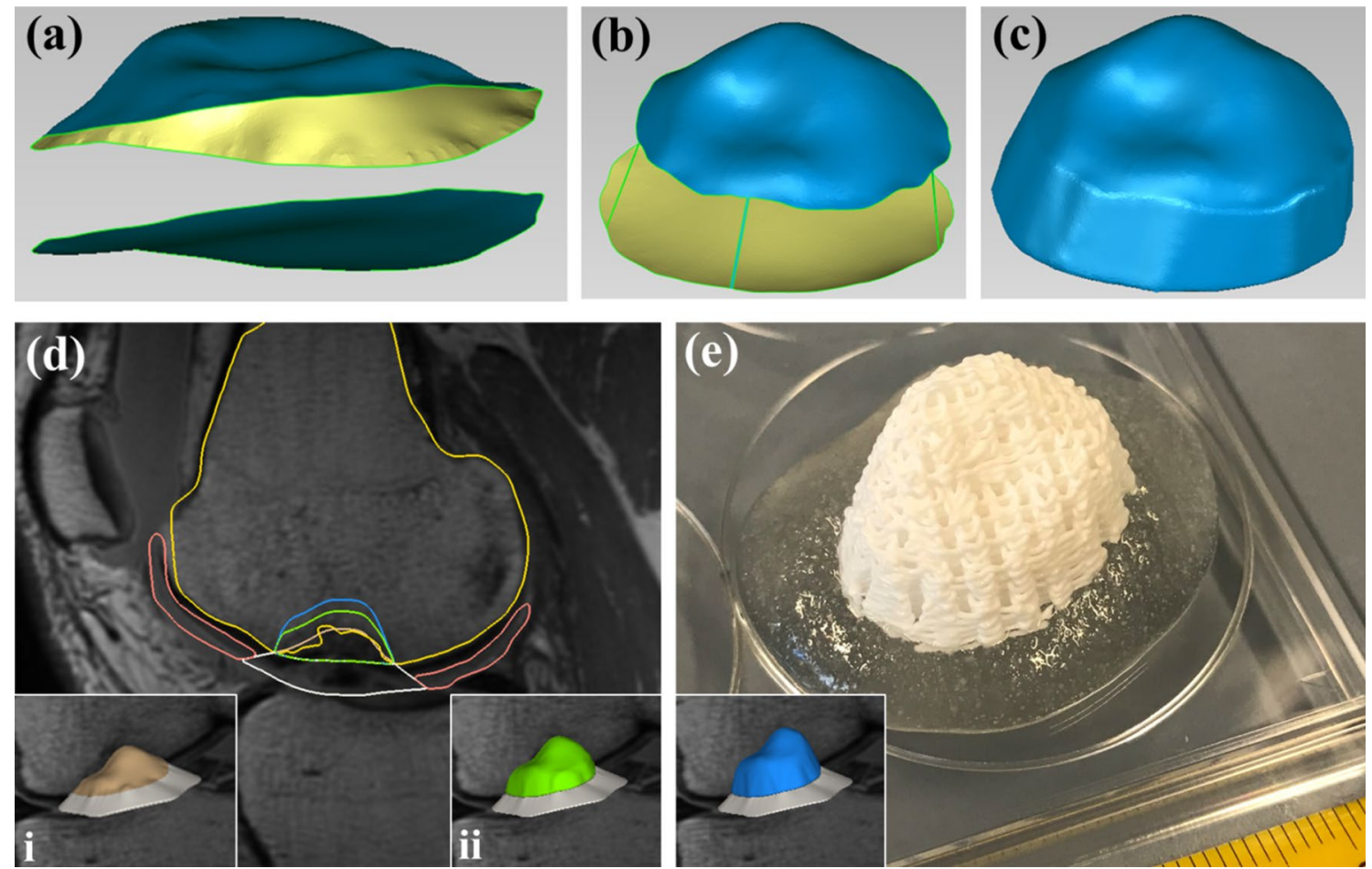

Fig. 6 Calculation of an internal "offset" to increase the inner implant size, enabling fixation of the implant via the conventional press-fit technique. a-c Offset generation process based on originally smoothed geometry: selection of the internal surface of the bone phase, moving by respective distance, and closing the contour again. d Sagittal view of the segmented defected FC region (yellow), the segmented AC phase (red), the AC phase of the implant (gray),

a highly potent tool [39], since cartilage tissue is not clearly visible in CT images. Recently, MRI data have also been shown to be beneficial for the design of non-implantable medical devices and demonstrators $[1,6]$ for various medical fields. So far, to the best of our knowledge, no study has used such data to generate an implant design and an actual 3D construct by means of 3D multichannel plotting under mild conditions. The most significant difference compared to previous segmentation and reproduction of human anatomical structures or any anthropomorphic models from MRI data is the condition that in the case of defected tissue, the geometry that needs to be designed does not actually exist anymore, i.e., it needs to be completed without complete knowledge of the structure of the lost tissue. Therefore, consideration of outer geometries like the convex surface of the $\mathrm{AC}$ in this study plays a crucial role. The underlying pathology diagnosed via DICOM data was identified as a stage 3-OCD lesion according to classification by Guhl et al. [40], with an $\mathrm{AC}$ malacia and a clearly developing fibrous necrotic zone (Fig. 1) that might lead to a dislocating "flap lesion" later. However, surgical intervention was required for that patient. The approach presented here is meant to target lesions above a size of ca. $2.5-3 \mathrm{~mm}^{2}$. Instead of using autologous bone and the smoothed bone phase geometries of varying offsets (beige: $0.3 \mathrm{~mm}$, green: $3 \mathrm{~mm}$, blue: $5 \mathrm{~mm}$ ); insets: biphasic implant design using bone phase depth offset of $0.3 \mathrm{~mm}$ (i) or $3.0 \mathrm{~mm}$ (ii). e 3D plotted biphasic implant structure of algMC and CPC with a bone phase depth offset of $5.0 \mathrm{~mm}$ and a CPC strand distance of $1.6 \mathrm{~mm}$, scale in $\mathrm{mm}$; inset: virtual biphasic implant design with an increased bone phase size (blue)

and an additional cartilage reconstruction or an autologous osteochondral plug, this study aimed to combine these tissue layers in one highly patient-specific multilayered implant. Besides the technical, design-related aspects, the central characteristics for the success of the presented concept were defining general requirements for a multilayered, osteochondral implant, evaluating the implant geometry, and allowing for a certain degree of flexibility for clinicians.

The challenge here was to overcome certain limitations of conventional autologous osteochondral grafts. A common limitation of recent approaches has been the lack of precise consideration of patient-specific defect geometry and anatomy. AM and bioprinting techniques that enable the precise reproduction of anatomical shapes play an increasingly important role in the generation of osteochondral tissue substitutes [29, 41]. With the use of MRI data for segmentation and a specific workflow (Fig. 2), these issues can be overcome [1].

The precise representation of the defect-facing geometry adjacent to the remaining SCB is not highly relevant to clinical performance since the remaining bone beneath the affected tissue is expected to be less stiff in a diseased state, so that by press-fitting the implant into the defect site, the 


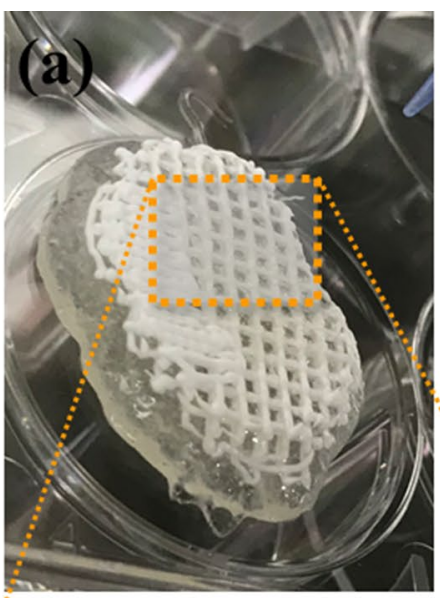

(b)
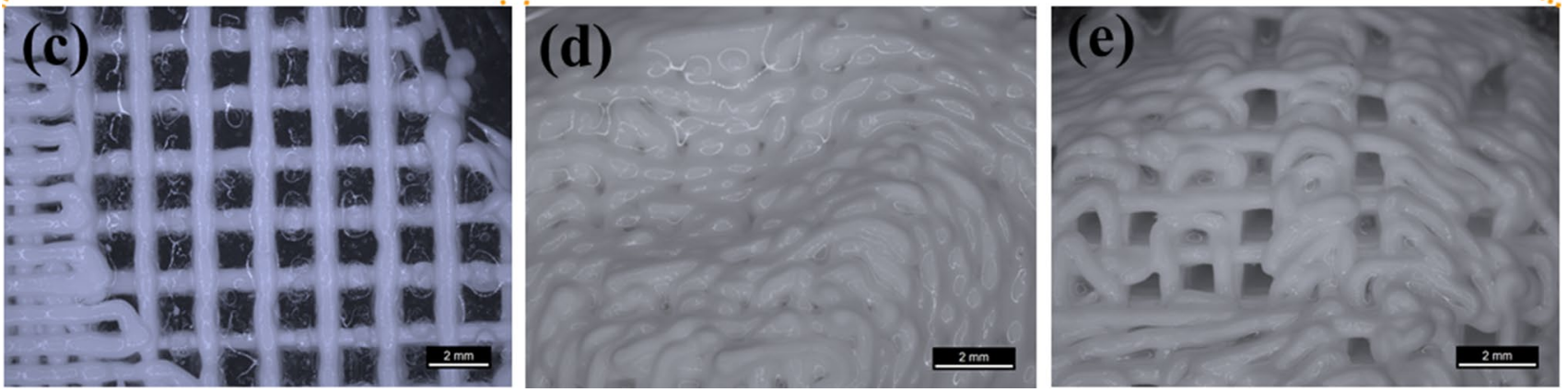

Fig. 7 Internal design criteria as essential characteristics for clinical performance. a An interwoven, biphasic zone during plotting. b In the sliced model of the cartilage phase, several layers of an interwoven network zone were considered. Light microscopic images of $\mathbf{c}$ the interwoven algMC $+\mathrm{CPC}$ zone, $\mathbf{d}$ a bone zone with low pore diameter (CPC strand distance set to $1.0 \mathrm{~mm}$ ), and $\mathbf{e}$ a larger pore diameter (CPC strand distance set to $1.6 \mathrm{~mm}$ )

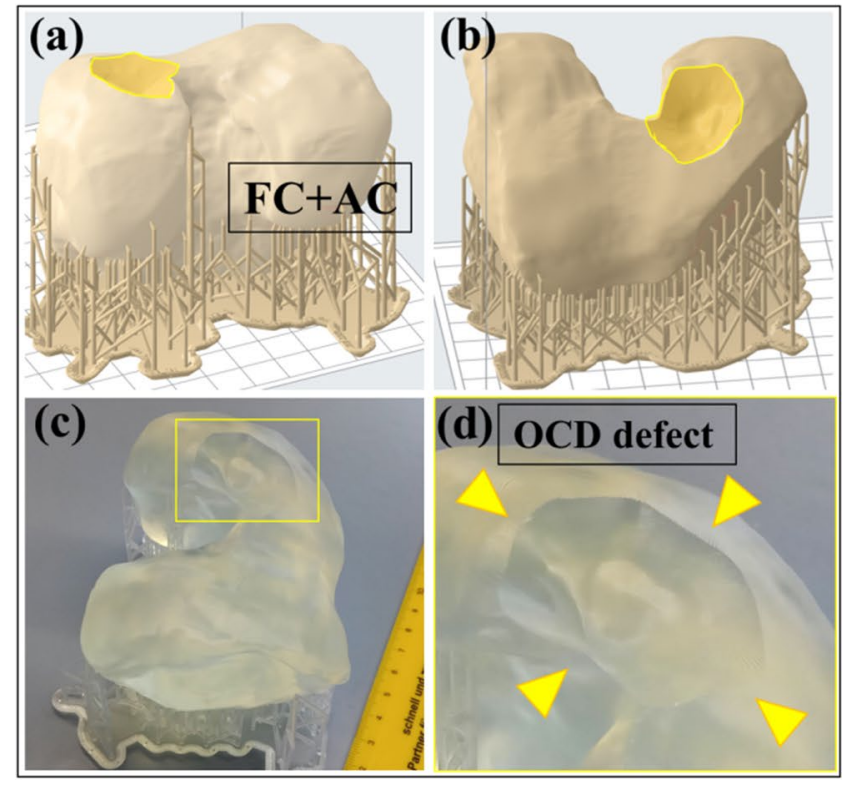

Fig. 8 Stereolithographic fabrication of a 3D model of the lesioned femoral condyles (FC) combined with the articular cartilage (AC). $\mathbf{a} / \mathbf{b}$ Virtual model of the printable geometry with support structures; lesioned site highlighted in yellow. c/d Resin 3D model after fabrication and post-curing; lesioned site highlighted in yellow (d, arrows),

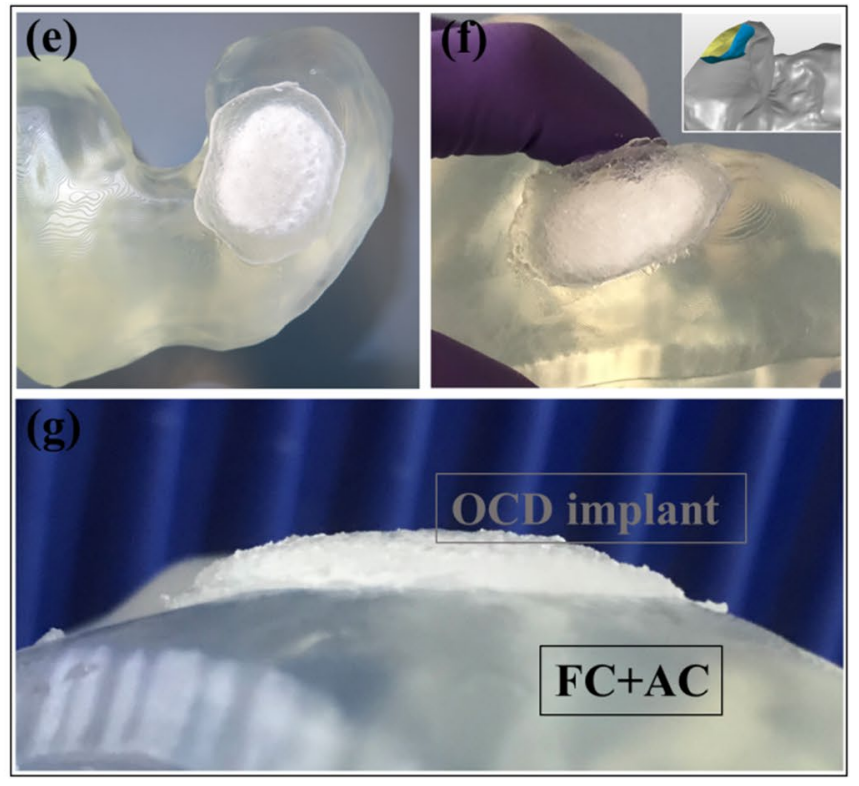

scale in $\mathrm{cm}$. e/f Fitting of the biphasic algMC + CPC implant from top (e) and side (f) views. g Outer convex implant geometry covers the convex shape of the lesioned femoral condyle with the lesioned AC surface 
Fig. 9 Design and generation of an individual implantation device adapter. a Virtual model of an individual adapter (gray, with $\mathrm{A}=$ anterior, $\mathrm{P}=$ posterior for spatial orientation during surgery) that fits onto the implant geometry (green outline) to replace damaged $\mathrm{AC}$ (red) and FC (yellow); inset: shape of the adapter tip which corresponds to the convex outer shape of the implant. b SLA-fabricated, resin-based fixation adapter; inset: removable support structures after SLA process, scales in $\mathrm{mm}$. c Attaching the individual fixation adapter to the surgical plunger shaft applied to fix the implant via the press-fit technique, that d fits onto the generated patientspecific implant for fixation
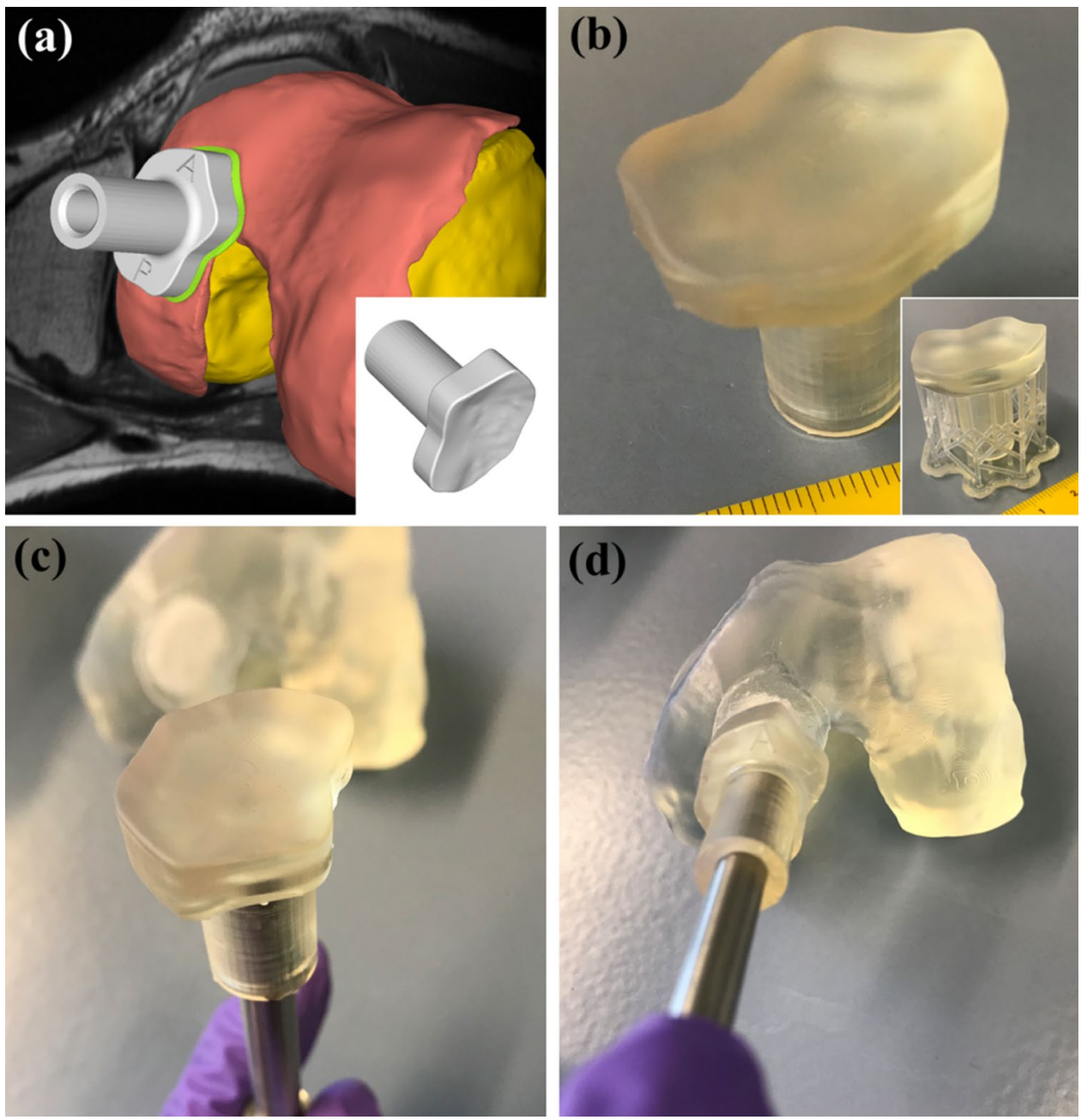

implant would be replacing some of that tissue during the implantation procedure. While that shape, therefore, might not be of major interest, the outer geometry certainly is: the AC tissue was identified as mostly intact, without a visible lesion. However, the tissue would have to be removed during surgical intervention, in order to access the lesioned bone site. Therefore, based on the MRI data, we virtually removed the covering $\mathrm{AC}$ zone with overhanging lateral edges, and later replaced it with the cartilage phase of the implant (Fig. 4b). The most crucial aspect here is the successful restoration of the convex outer surface line of the AC substitute (Figs. $5 \mathrm{f}$ and $8 \mathrm{~g}$ ). For better fusion of the material phases, and as a potential calcified cartilage layer, we included an interwoven zone (Fig. 7c) with a thickness similar to that of the calcified cartilage layer in vivo. This particular zone can be included as a non-individual even surface or as an additional zone with an individualized geometry. With a fairly flexible workflow and implant design, the surgeon would also have several options to choose from spontaneously during surgery, based on the pathological situation in vivo. The main flexible characteristics would include the size (offset) and degree of porosity of the CPC phase for the bone-replacing layer (Figs. 6 and 7). In addition to being adjustable for the z-direction, this size can also be adjusted to lateral directions by an additional offset value when needed. The generated implant fitted quite well into a SLA-fabricated defect site model. This defect model was based on the same segmentation as that of the implant. This primarily ensured the suitability of the material combination, the $3 \mathrm{D}$ plotting process, and the post-processing for precise implant fabrication. Therefore, the defect model was fabricated by SLA, a very precise but different AM method that has been described for that purpose in oral surgery [7], but does not allow the generation of biodegradable multilayered implants. The fabricated FC defect site model merely resembles the geometric shape of the defect and does not fully consider the mechanical circumstances of an actual implantation in vivo. However, the application shows the valuable potential of SLA-processed material for geometric anatomical models [7]. Due to the fact that we presented our workflow based on a real patient case and did not plan to include in vivo evaluation, a fitting of the designed tissue 
substitute into the actual defect site was not part of the scope or aims of the study.

\section{Selecting materials with regenerative potential in vitro and in vivo}

Hydrogel inks can be applied as biomaterial inks or bioinks, with or without the encapsulation of cells [42]. However, many common materials suitable for bioinks do not allow the stable stacking of a larger number of extruded layers that would be sufficient for the required clinically relevant dimensions, or do so only after application of additional technical stabilization strategies [43]. Applying the material blend algMC enables the formation of volumetric structures and, in addition, allows the incorporation of cells [29, 38] or specific growth factors [44] that can attract or support cells and ingrowth of blood vessels. The degree of porosity of CPC can play an essential role in the success rate of an implant, as examined for maxillofacial defects in rats [32]. In this study, we suggested two different pore sizes of CPC, arising from strand distances of $1.0 \mathrm{~mm}$ and $1.6 \mathrm{~mm}$ (Fig. 7d and 7e). The pore size impacts the mechanical stability (brittleness) of the bone zone and the invasion behavior of surrounding cells and capillaries in vivo. Furthermore, tuning the CPC spacing allows for some flexibility of the SCB-facing shape, which might be helpful during surgical press-fit implantation. Although the specific CPC used in this study is clinically approved and alginate has shown good biocompatibility before, future in vivo evaluation is needed: We suggest an initial validation of fitting geometries in a cadaveric OCD defect model [45], while pre-clinical evaluation in rabbits or sheep would be needed prior to possible clinical evaluation [46, 47].

\section{Consideration of implantation strategies}

The pores ensure omni-directional integration with surrounding cells and vessels, as well as an influx of oxygen and nutrients and a supply of various essential growth factors contained in the surrounding blood after scraping or microfracturing the underlying remaining bone tissue. With respect to the mechanical properties, the structure after degradation/resorption will be expected to resemble the porosity of native cancellous bone [48]. Prior to implantation, the encapsulation of cells inside the hydrogel (as a bioink), which is possible in combination with CPC $[29,38]$, or preseeding the CPC [32] phase, could be a valuable option to improve or accelerate regeneration. The effect will need to be investigated. Many options could be added and modified for the implant design. This includes the potential consideration of certain cartilage layers of different material densities and with different fiber orientations [49], which might be technically achievable but not beneficial. Fiber orientation cannot be detected via MRI. Moreover, in the interdisciplinary yet rather academic approach presented here, over-eng $i$ neering, the pure technical adjustment of additional parameters without certain clinical relevance, should be avoided.

However, an essential aspect that needs to be considered is the possibility of fixing the implant in the defect site, which directly influences the on-site integration and post-surgery performance [50]. The interlock between the hydrogel and cement phase is quite strong with an additional interwoven network zone (Fig. 7).

Therefore, conventional press-fit implantation would not harm the integrity of the algMC-to-CPC interface [51] as such (the mechanical properties of the interface have been sufficiently described before [38]). However, depending on the architecture and porosity of the rather brittle CPC phase, the well-ordered structure of the hardened CPC strands might be impaired; while fixation of the implant with a conventional surgical plunger might damage the hydrogel surface of the implant. AlgMC and other hydrogel blends used as a cartilage zone would require a relatively gentle implantation procedure. To avoid rupture, we designed an individually shaped fixation tool (Fig. 9) as an adapter to the conventionally applied plunger, based on an autoclavable resin. To further ensure the integrity of the hydrogel, insertion of fine reinforcing microfibers into the hydrogel could be helpful [52]. As a biological bonding agent, fibrin tissue glue could be applied as an optional addition to the press-fit approach [53, 54].

\section{Necessity for a novel bidirectional CAD/CAM workflow}

In addition to the implant design and generation, we presented a novel strategy for an optimized, bidirectional CAD/ CAM workflow (Fig. 4a): The main advantage of the CTinA software tool is the bidirectional interface $[3,37]$ from the MRI data to the CAD system, which allows full freedom for reversibility and correction of previous editing steps. Thus, no information is lost during additional import/export steps between different software tools. Furthermore, it allows the visualization of different phases of the implant design, and therefore simultaneous editing of 3D-rendered CT/MRI data, tomographs, and segmented $s t l$ models of the implant (Figs. 3-5). This aspect is beneficial for the reliability of the implant design and the documentation of the design process, but above all, simplifies, accelerates, and improves the quality of the modeling. The use of MRI instead of CT data allows clear separation of different soft and hard tissues. Advancing developments of high-field MRI scanners with improved resolution also have great potential to limit the challenges of image processing, while the image contrast already provides clear advantages over other imaging techniques [6]. However, thresholding the gray values correctly 
can be a complex and time-consuming step. For a pure bone implant, therefore, usually CT data would be the preferred dataset. This difficulty was avoided with the presented solution of a software interface that includes appropriate modeling tools but does not require a several-step workflow covering separated transfer and conversion via segmentation and model generation. This also allows inclusion of reference points to serve as rotation axes, reference plains, or boundary lines. Therefore, an accurate representation of the defect geometry and the respective tissue substitutes was possible. These tissue substitutes can be freely adjusted after completing the parametric modeling [55], ensuring a flexible and transferrable workflow.

\section{Conclusions}

The development of this CAD/CAM workflow for a biphasic, zonal OCD implant involved close communication and collaboration between life scientists, mechanical engineers, and clinicians in order to explore the relevance and possible adjustment of certain implant properties and geometries. We described and demonstrated for the first time, to the best of our knowledge, a fully integrated approach for an MRIguided multilayered implant design that can be realized by extrusion printing, using custom-adjusted tools for commercially available software. The implant design allows a certain amount of flexibility for the clinical requirements; as a model system, we used a biphasic osteochondral implant based on algMC as a potential bioink to replace the AC layer, and 3D plotted CPC with high shape fidelity to restore the subchondral bone portion. The results showed perfect geometric compatibility with an SLA-fabricated defect model. The visualization of certain levels of the implant design process: 1) CT/MRI data, 2) segmented defect site models, and 3) implant design, by a novel use of the CTinA tool, will certainly be helpful to overcome many clinical challenges in the future. Looking to the future, this procedural concept can be transferred to other patients and other clinical situations, as well as other types of tissue defects or damaged organs diagnosed by MRI. Therefore, this study of one clinical case demonstrated a synergistic collaboration of engineering, biomedicine, and clinical medicine to develop concepts and technical solutions with the potential for real clinical application in medium and large defects $\left(>3 \mathrm{~cm}^{2}\right)$. The clinical performance of the generated implants and the presented interdisciplinary process will need to be evaluated as well. This proof-of-concept study opens up new possibilities for the design of individual OCD implants but also for other applications in the field of anatomically shaped tissue models, anthropomorphic phantom generation, or 3D organ models for tumor diagnosis, patient information, and surgical training.
Supplementary Information The online version contains supplementary material available at https://doi.org/10.1007/s42242-021-00153-4.

Acknowledgements The authors would like to thank the European Social Fund (ESF) and the Free State of Saxony for financial support in the course of the Young Researchers' Group IndivImp. Furthermore, the authors acknowledge Sophie Dani and Max von Witzleben for their technical support with the preparation of plottable $3 m f$ files and SLA printing.

Author contributions All authors contributed to this manuscript and gave permission for the final version of the publication. DK curated the data and contributed to conceptualization, formal analysis, methodology, investigation, and writing - original draft; PS curated the data and contributed to formal analysis, software, methodology, and writing - original draft; HB contributed to conceptualization, formal analysis, resources, and writing-reviewing and editing; TA contributed to formal analysis, methodology, and writing-reviewing and editing; LM contributed to formal analysis, software, methodology, and writing-reviewing and editing; JL contributed to resources, validation, supervision, and writing - reviewing and editing; SH contributed to methodology, software, and writing - reviewing and editing; AL, RS, and MG administrated the project, helped in supervision, acquired funding, and contributed to writing - reviewing and editing.

Funding Open Access funding enabled and organized by Projekt DEAL.

Data availability The data that support the findings of this study are available from the corresponding author upon reasonable request.

\section{Declarations}

Conflict of interest The authors declare that there is no conflict of interest.

Ethical approval Anonymized datasets from one patient with osteochondritis dissecans were provided with permission for researchrelated use in this study by the University Center of Orthopaedic, Trauma and Plastic Surgery, University Hospital Carl Gustav Carus, Technische Universität Dresden, Germany. All procedures followed were in accordance with the ethical standards and regulations of the responsible committees of the University Hospital Carl Gustav Carus, Technische Universität Dresden.

Consent for publication All authors gave permission for this publication.

Open Access This article is licensed under a Creative Commons Attribution 4.0 International License, which permits use, sharing, adaptation, distribution and reproduction in any medium or format, as long as you give appropriate credit to the original author(s) and the source, provide a link to the Creative Commons licence, and indicate if changes were made. The images or other third party material in this article are included in the article's Creative Commons licence, unless indicated otherwise in a credit line to the material. If material is not included in the article's Creative Commons licence and your intended use is not permitted by statutory regulation or exceeds the permitted use, you will need to obtain permission directly from the copyright holder. To view a copy of this licence, visit http://creativecommons.org/licenses/by/4.0/. 


\section{References}

1. Haleem A, Javaid M (2018) Role of CT and MRI in the design and development of orthopaedic model using additive manufacturing. J Clin Orthop Trauma 9(3):213-217. https://doi.org/10. 1016/j.jcot.2018.07.002

2. Mika L, Sembdner P, Heerwald S et al (2020) Konstruktion eines individuellen Knieimplantates für die Fertigung mittels EBM. Konstruktion für die Addit Fertigung 2019:57-71 (in German). https://doi.org/10.1007/978-3-662-61149-4_5

3. Sembdner P, Holtzhausen S, Schöne C et al (2013) Additional methods to analyze computer tomography data for medical purposes and generatively produced technical components, digital product and process development systems: IFIP TC 5 International Conference. New Prolamat 2013:221-229. https://doi.org/ 10.1007/978-3-642-41329-2_23

4. Ni J, Ling H, Zhang S et al (2019) Three-dimensional printing of metals for biomedical applications. Mater Today Bio 3(2019):100024. https://doi.org/10.1016/j.mtbio.2019.100024

5. Javaid M, Haleem A (2018) Additive manufacturing applications in orthopaedics: a review. J Clin Orthop Trauma 9(3):202-206. https://doi.org/10.1016/j.jcot.2018.04.008

6. Ripley B, Levin D, Kelil T et al (2017) 3D printing from MRI data: harnessing strengths and minimizing weaknesses. J Magn Reson Imaging 45:635-645. https://doi.org/10.1002/jmri. 25526

7. Mehra P, Miner J, D'Innocenzo R et al (2011) Use of 3-d stereolithographic models in oral and maxillofacial surgery. J Maxillofac Oral Surg 10:6-13. https://doi.org/10.1007/ s12663-011-0183-3

8. Sun W, Starly B, Nam J et al (2005) Bio-CAD modeling and its applications in computer-aided tissue engineering. Comput-Aid Des 37:1097-1114. https://doi.org/10.1016/j.cad.2005.02.002

9. Mishinov SV, Stupak VV, Koporushko NA et al (2018) Titanium patient-specific implants in reconstructive neurosurgery. Biomed Eng 52:152-155. https://doi.org/10.1007/s10527-018-9802-3

10. Woodruff MA, Hutmacher DW (2010) The return of a forgotten polymer-polycaprolactone in the 21st century. Progr Polym Sci 35:1217-1256. https://doi.org/10.1016/j.progpolymsci.2010.04. 002

11. Ahlfeld T, Köhler T, Czichy C et al (2018) A methylcellulose hydrogel as support for 3D plotting of complex shaped calcium phosphate scaffolds. Gels 4(3):68. https://doi.org/10.3390/gels4 030068

12. Malda J, Visser J, Melchels FP et al (2013) 25th anniversary article: engineering hydrogels for biofabrication. Adv Mater 25:5011-5028. https://doi.org/10.1002/adma.201302042

13. Obedian RS, Grelsamer RP (1997) Osteochondritis dissecans of the distal femur and patella. Clin Sports Med 16:157-174. https:// doi.org/10.1016/S0278-5919(05)70012-0

14. Hefti F, Beguiristain J, Krauspe R et al (1999) Osteochondritis dissecans: a multicenter study of the European Pediatric Orthopedic Society. J Pediatr Orthop B 8:231-245

15. Peterson L, Minas T, Brittberg M et al (2003) Treatment of osteochondritis dissecans of the knee with autologous chondrocyte transplantation: results at two to ten years. J Bone Joint Surg 85-A(Suppl 2):17-24. https://doi.org/10.2106/00004623-20030 0002-00003

16. Lützner J, Mettelsiefen J, Günther KP et al (2007) Therapie der Osteochondrosis dissecans des Kniegelenks. Orthopade 36:871879 (in German). https://doi.org/10.1007/s00132-007-1130-3

17. Lomax A, Calder J (2016). Retrograde drilling for the treatment of osteochondral lesions in the ankle. In: Randelli P, Dejour D, van Dijk C, Denti M, Seil R (eds) Arthroscopy: 1023-1031. https:// doi.org/10.1007/978-3-662-49376-2_85
18. Erggelet C, Steinwachs MR, Reichelt A (2000) The operative treatment of full thickness cartilage defects in the knee joint with autologous chondrocyte transplantation. Saudi Med J 21:715-721

19. Glasbrenner J, Petersen W, Raschke MJ et al (2020) Matrix-augmented bone marrow stimulation with a polyglycolic acid membrane with hyaluronan vs microfracture in local cartilage defects of the femoral condyles: a multicenter randomized controlled trial. Orthop J Sports Med 8(5):2325967120922938. https://doi.org/10. $1177 / 2325967120922938$

20. Niemeyer P, Becher C, Brucker PU et al (2018). Stellenwert der matrixaugmentierten Knochenmarkstimulation in der Behandlung von Knorpelschäden des Kniegelenks: Konsensusempfehlungen der AG Klinische Geweberegeneration der DGOU, Z. Orthop. Unfall. 156: 513-532 (in German). https://doi.org/10. 1055/a-0591-6457.

21. Sacolick DA, Kirven JC, Abouljoud MM et al (2019) The treatment of adult osteochondritis dissecans with autologous cartilage implantation: a systematic review. J Knee Surg 32:1102-1110. https://doi.org/10.1055/s-0038-1675568

22. Deng Z, Jin J, Zhao J et al (2016) Cartilage defect treatments: with or without cells? Mesenchymal stem cells or chondrocytes? Traditional or matrix-assisted? A systematic review and meta-analyses. Stem Cells Int 2016:9201492. https://doi.org/10.1155/2016/9201492

23. Duchow J, Hess T, Kohn D (2000) Primary stability of press-fitimplanted osteochondral grafts. Influence of graft size, repeated insertion, and harvesting technique. Am J Sports Med 28:24-27. https://doi.org/10.1177/03635465000280011601

24. Lee DW, Kim JG, Ha JK et al (2016) Simultaneous osteoperiosteal autologous iliac crest graft and lateral meniscus allograft transplantation for osteochondral lesion with bony defect and lateral discoid meniscus tear. Knee Surg Relat Res 28:165-171. https:// doi.org/10.5792/ksrr.2016.28.2.165

25. Hinzpeter J, Zamorano A, Barahona M et al (2019) Treatment of osteochondritis dissecans of the knee with autologous iliac bone graft and hyaluronic acid scaffold. Knee Surg Relat Res 31:143146. https://doi.org/10.5792/ksrr.18.053

26. Langer R, Vacanti JP (1993) Tissue engineering. Science 260:920-926. https://doi.org/10.1126/science.8493529

27. Martín AR, Patel JM, Zlotnick HM et al (2019) Emerging therapies for cartilage regeneration in currently excluded "red knee" populations. npj Regen Med 4:12. https://doi.org/10.1038/ s41536-019-0074-7

28. Paunipagar BK, Rasalkar D (2014) Imaging of articular cartilage. Indian J Radiol Imaging 24:237-248. https://doi.org/10. 4103/0971-3026.137028

29. Kilian D, Ahlfeld T, Akkineni AR et al (2020) 3D Bioprinting of osteochondral tissue substitutes - in vitro-chondrogenesis in multi-layered mineralized constructs. Sci Rep 10:8277. https:// doi.org/10.1038/s41598-020-65050-9

30. GhavamiNejad A, Shammakhi NA, Wu XY et al (2020) Crosslinking strategies for 3D bioprinting of polymeric hydrogels. Small 16:e2002931. https://doi.org/10.1002/smll.202002931

31. Ahlfeld T, Akkineni AR, Förster Y et al (2017) Design and fabrication of complex scaffolds for bone defect healing: combined $3 \mathrm{D}$ plotting of a calcium phosphate cement and a growth factorloaded hydrogel. Ann Biomed Eng 45:224-236. https://doi.org/ 10.1007/s10439-016-1685-4

32. Korn P, Ahlfeld T, Lahmeyer F et al (2020) 3D printing of bone grafts for cleft alveolar osteoplasty - in vivo evaluation in a preclinical model. Front Bioeng Biotechnol 8:217. https://doi.org/10. 3389/fbioe.2020.00217

33. Lode A, Meissner K, Luo Y et al (2014) Fabrication of porous scaffolds by three-dimensional plotting of a pasty calcium phosphate bone cement under mild conditions. J Tissue Eng Regen Med 8:682-693. https://doi.org/10.1002/term.1563 
34. Heinemann S, Rössler S, Lemm M et al (2013) Properties of injectable ready-to-use calcium phosphate cement based on waterimmiscible liquid. Acta Biomater 9:6199-6207. https://doi.org/10. 1016/j.actbio.2012.12.017

35. Reitmaier S, Kovtun A, Schuelke J et al (2018) Strontium(II) and mechanical loading additively augment bone formation in calcium phosphate scaffolds. J Orthop Res 36:106-117. https://doi.org/10. 1002/jor.23623

36. Baranowski A, Klein A, Ritz U et al (2018) Evaluation of bone sialoprotein coating of three-dimensional printed calcium phosphate scaffolds in a calvarial defect model in mice. Materials 11(11):2336. https://doi.org/10.3390/ma11112336

37. Hofmann D, Sembdner P, Holtzhausen S et al (2018). Approach for using CT data in product development processes. 8th Conference on Industrial Computed Tomography (iCT) 2018 2018-02

38. Ahlfeld T, Doberenz F, Kilian D et al (2018) Bioprinting of mineralized constructs utilizing multichannel plotting of a self-setting calcium phosphate cement and a cell-laden bioink. Biofabrication 10:45002. https://doi.org/10.1088/1758-5090/aad36d

39. Quatman CE, Quatman-Yates CC, Schmitt LC et al (2012) The clinical utility and diagnostic performance of MRI for identification and classification of knee osteochondritis dissecans. J Bone Joint Surg Am 94(11):1036-1044. https://doi.org/10.2106/ JBJS.K.00275

40. Guhl JF (1979) Arthroscopic treatment of osteochondritis dissecans: preliminary report. Orthop Clin North Am 10:671-683

41. Daly AC, Freeman FE, Gonzalez-Fernandez T et al (2017) 3D Bioprinting for cartilage and osteochondral tissue engineering. Adv Healthcare Mater 6(22):1700298. https://doi.org/10.1002/ ADHM.201700298

42. Groll J, Burdick JA, Cho DW et al (2018) A definition of bioinks and their distinction from biomaterial inks. Biofabrication 11:13001. https://doi.org/10.1088/1758-5090/aaec52

43. Kilian D, Ahlfeld T, Akkineni AR et al (2017) Three-dimensional bioprinting of volumetric tissues and organs. MRS Bull 42:585592. https://doi.org/10.1557/mrs.2017.164

44. Ahlfeld T, Schuster FP, Förster Y et al (2019) 3D plotted biphasic bone scaffolds for growth factor delivery: biological characterization in vitro and in vivo. Adv Healthcare Mater 8(7):1801512. https://doi.org/10.1002/ADHM.201801512

45. Hoser C, Bichler O, Bale R et al (2004) A computer assisted surgical technique for retrograde autologous osteochondral grafting in talar osteochondritis dissecans (OCD): a cadaveric study. Knee
Surg Sports Traumatol Arthrosc 12:65-71. https://doi.org/10. 1007/s00167-003-0394-3

46. Dias IR, Viegas CA, Carvalho PP (2018) Large animal models for osteochondral regeneration. Adv Exp Med Biol 1059:441-501. https://doi.org/10.1007/978-3-319-76735-2_20

47. Meng X, Ziadlou R, Grad S et al (2020) Animal models of osteochondral defect for testing biomaterials. Biochem Res Int 2020:9659412. https://doi.org/10.1155/2020/9659412

48. Polo-Corrales L, Latorre-Esteves M, Ramirez-Vick JE (2014) Scaffold design for bone regeneration. J Nanosci Nanotechnol 14:15-56. https://doi.org/10.1166/jnn.2014.9127

49. Mansfield JC, Winlove CP, Moger J et al (2008) Collagen fiber arrangement in normal and diseased cartilage studied by polarization sensitive nonlinear microscopy. J Biomed Opt 13:44020. https://doi.org/10.1117/1.2950318

50. Mancini IAD, Vindas Bolaños RA, Brommer H et al (2017) Fixation of hydrogel constructs for cartilage repair in the equine model: a challenging issue. Tissue Eng Part C Methods 23:804814. https://doi.org/10.1089/ten.TEC.2017.0200

51. Agneskirchner JD, Brucker P, Burkart A et al (2002) Large osteochondral defects of the femoral condyle: press-fit transplantation of the posterior femoral condyle (MEGA-OATS). Knee Surg Sports Traumatol Arthroscopy 10:160-168. https://doi.org/10. 1007/s00167-001-0259-6

52. Visser J, Melchels FPW, Jeon JE et al (2015) Reinforcement of hydrogels using three-dimensionally printed microfibers. Nat Commun 6:6933. https://doi.org/10.1038/ncomms7933

53. Shah MA, Ebert AM, Sanders WE (2002) Fibrin glue fixation of a digital osteochondral fracture: case report and review of the literature. J Hand Surg 27:464-469. https://doi.org/10.1053/jhsu. 2002.32957

54. Filardo G, Drobnic M, Perdisa F et al (2014) Fibrin glue improves osteochondral scaffold fixation: study on the human cadaveric knee exposed to continuous passive motion. Osteoarthr Cartil 22:557-565. https://doi.org/10.1016/j.joca.2014.01.004

55. George SP, Saravana Kumar G (2013) Patient specific parametric geometric modelling and finite element analysis of cementless hip prosthesis. Virt Phys Prototyp 8:65-83. https://doi.org/10.1080/ 17452759.2012.755654 